\title{
Scanning Brain Networks with Micro-CT
}

\author{
Ryuta Mizutani, ${ }^{\text {* }}$ Rino Saiga, ${ }^{1}$ Susumu Takekoshi, ${ }^{2}$ Makoto Arai, ${ }^{3}$ Akihisa Takeuchi, ${ }^{4}$ and \\ Yoshio Suzuki ${ }^{4}$ \\ ${ }^{1}$ Department of Applied Biochemistry, Tokai University, Hiratsuka, Kanagawa 259-1292, Japan \\ ${ }^{2}$ Tokai University School of Medicine, Isehara, Kanagawa 259-1193, Japan \\ ${ }^{3}$ Tokyo Metropolitan Institute of Medical Science, Setagaya, Tokyo 156-8506, Japan \\ ${ }^{4}$ Japan Synchrotron Radiation Research Institute (JASRI/SPring-8), Sayo, Hyogo 679-5198, Japan \\ *ryuta@tokai-u.jp
}

\section{Introduction}

A wide variety of brain functions including cognition, verbal ability, and decision making are performed by the synergy of a huge number of neurons configured in a threedimensional (3D) network in brain tissue. The first step to understanding how the brain functions is to analyze this $3 \mathrm{D}$ structure. Because neurons exert their functions by interacting with each other, a number of neurons should be visualized simultaneously to illustrate the functional mechanisms of the brain. Although structural studies of brain tissues have been reported, the structures of these tissues, which are composed of many neurons, are difficult to comprehend. This is because there is a lack of quantitative descriptions of neuronal networks, which should be represented with 3D Cartesian coordinates, rather than a 3D distribution of intensities.

This article reports the structures of brain tissues belonging to humans [1] and the fruit fly Drosophila melanogaster [2] that were ascertained using X-ray tomographic microscopy, a microscopic version of medical computed tomography (CT). In order to analyze the brain network, the structure of each neuron should be described in terms of 3D Cartesian coordinates by building skeletonized models of neurons. The structural components of the fly brain network were classified on the basis of their 3D structures. This classified model allowed identification of anatomical segments. The network of human brain tissue indicated that the neurons form feedback loops. Such feedback loops can be viewed as canonical elements in brain circuits. (a)

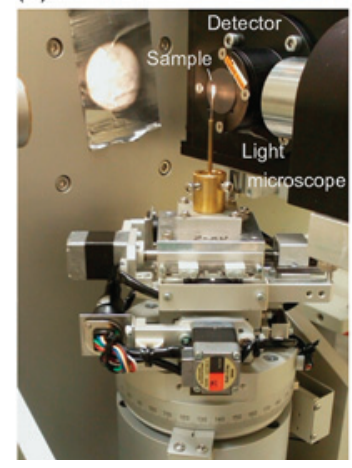

(b)

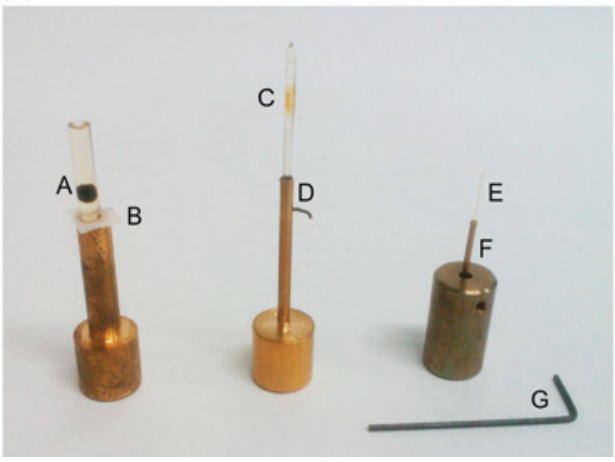

\section{Materials and Methods}

Contrasting brain tissues. The transparency of biological tissue to hard $\mathrm{X}$ rays enables radiographic analysis of tissue structures. Soft tissues are composed of light elements, which produce little contrast in a hard X-ray image. In clinical diagnosis, luminal structures of a living body are visualized by using X-ray contrast media. These contrast media contain high-atomic-number (high- $Z$ ) elements that attenuate $\mathrm{X}$ rays efficiently. Tissue-staining methods using high- $Z$ elements, such as Golgi impregnation and reduced-silver impregnation, are known as conventional techniques for the histology of brain tissues. In this study, the tissue structures were visualized with these impregnation methods.

Post-mortem human cerebral tissues were collected with informed consent from the legal next of kin using protocols approved by ethical committees of organizations related to this study. The frontal cortex tissues were subjected to Golgi impregnation, as described previously [1]. Neurons were visualized with silver deposits in dendrites, axons, and cell bodies with this method.

Wild-type fruit flies Drosophila melanogaster Canton-S (Drosophila Genetic Resource Center, Kyoto Institute of Technology, Japan) were raised on standard cornmealmolasses fly food and kept at $20^{\circ} \mathrm{C}$. Adult fly brains, also referred to as the cephalic ganglion, were dissected and subjected to modified reduced-silver impregnation, as described previously [2]. It has been shown that neurons were visualized with metal gold particles derived from the aurate (c)

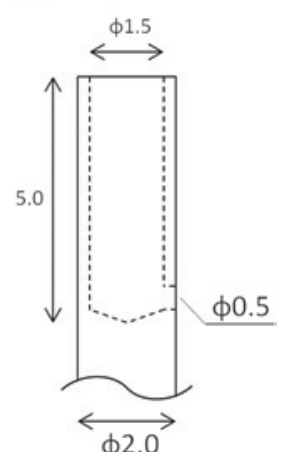

Figure 1: (a) X-ray tomographic microscopy at BL20XU of SPring-8. The incident X-ray beam comes from the left, and transmission images were acquired with the detector. (b) Samples for tomographic microscopy. Pellet sample (labeled A) was attached to the flat surface of the brass fitting using double-stick tape B. Capillary sample C was inserted in clay stuffed in a hole of brass fitting $D$. Clay ejected from a side opening can be seen. Capillary sample $E$ sleeved with a brass tube F was fixed using a wrench G. (c) Schematic drawing of the upper end of the fitting D. An opening with a diameter of $0.5 \mathrm{~mm}$ was made for venting air and clay under pressure in the hole. toning reagent used in this method.

Tissue embedding. Tissue structures should be kept constant during the data collection. However, biological soft tissues themselves are not rigid and show deformations depending on ambient conditions. Therefore, the tissue samples were embedded in epoxy resin $[1,2]$ as one would do in the case of transmission electron microscopy.

Resin embedding was performed by transferring the tissue sample from an aqueous solution to first ethanol, then to $n$-butyl glycidyl ether, and finally to the resin. In the case of embedding a block sample with dimensions of $5 \times 5 \times 5 \mathrm{~mm}^{3}$, the 
Table 1: X-ray tomographic microscopy data collection conditions.

\begin{tabular}{|l|l|l|l|}
\hline Beamline & BL20XU & BL37XU & BL47XU \\
\hline X-ray optics & Simple projection & Fresnel zone plate optics & Fresnel zone plate optics \\
\hline X-ray energy $(\mathrm{keV})$ & 12 & 8 & 8 \\
\hline Pixel size & $\mathbf{a}$ & $60 \times 60 \mathrm{~nm}$ & $262 \times 262 \mathrm{~nm}$ \\
\hline Viewing field size (pixels) ${ }^{a}$ & $2,048 \times 2,048$ & $2,048 \times 2,048$ & $2,000 \times 700$ \\
\hline Viewing field size $(\mu \mathrm{m})^{\mathrm{a}}$ & $1,024 \times 1,024$ & $123 \times 123$ & $524 \times 183$ \\
\hline Rotation/frame (degrees) & 0.10 & 0.20 & 0.10 \\
\hline Exposure/frame $(\mathrm{ms})$ & 150 & 700 & 300 \\
\hline Frame/dataset & 1,800 & 900 & 1,800 \\
\hline Dataset collection time $(\mathrm{min})$ & 6 & 20 & 16 \\
\hline Spatial resolution & $1.0-1.2 \mu \mathrm{m}$ & $160-200 \mathrm{~nm}$ & $600-800 \mathrm{~nm}$ \\
\hline
\end{tabular}

awidth $\times$ height

tissue was soaked in $10 \mathrm{~mL}$ of $100 \% \mathrm{EtOH}$ for a few hours or overnight at room temperature $\left(20-25^{\circ} \mathrm{C}\right)$. This process was repeated three times in total. The tissue was then transferred to $10 \mathrm{~mL}$ of $n$-buthyl glycigylether and left for several hours or overnight at room temperature. This process was repeated twice in total. Subsequently, the tissue was soaked in a $2 \mathrm{~mL}$ aliquot of Petropoxy 154 (Burnham Petrographics, ID) epoxy resin overnight at $4^{\circ} \mathrm{C}$. The epoxy resin was degassed prior to use. This process was repeated twice in total. If the tissue floated to the surface of the resin even after two cycles of resin soaking, an additional soaking using another resin aliquot was performed.

Human brain tissues soaked in the resin were cut into rod shapes with widths of $0.3-0.5 \mathrm{~mm}$ under a stereomicroscope and transferred to a borosilicate glass capillary (W. Müller, Germany) filled with the resin. Larger tissue samples with widths of $1.5-3 \mathrm{~mm}$ were also prepared and embedded in resin pellets. The fly brain was embedded in a tiny resin drop using a nylon loop for protein crystallography (Hampton Research, CA). The samples were then kept at $90^{\circ} \mathrm{C}$ for $40-90 \mathrm{hr}$ for curing the resin.
Sample mounting. The resin-embedded samples were mounted on the sample stage using brass fittings (Figure 1a). Example samples are shown in Figure 1b. Pellet samples were attached to the flat surface of the brass fitting using epoxy glue or double-stick tape. Epoxy glue is preferable for high-resolution analysis. Capillary samples with outer diameters greater than $0.5 \mathrm{~mm}$ were inserted in clay stuffed in a hole made at the end of the brass fitting. Because the capillary insertion compresses the residual air in the hole and its re-expansion can cause sample drift during data collection, a minuscule side opening was made in the lateral face of the fitting (Figure 1c) to vent the air and clay. Capillaries with diameters less than $0.5 \mathrm{~mm}$ were sleeved with a brass tube using epoxy glue and secured with a setscrew. Sample mounting was finished at least 30 minutes before the data collection to stabilize the samples. For tomographic microscopy with resolutions higher than $200 \mathrm{~nm}$, the mounted samples should be placed near the sample stage as soon as possible in order to equilibrate their temperature with that of the apparatus.

Tomographicmicroscopy.Simple-projection tomographic microscopywasperformedattheBL20XUbeamlineoftheSPring-8 synchrotron radiation facility, as reported previously [1]. (a)

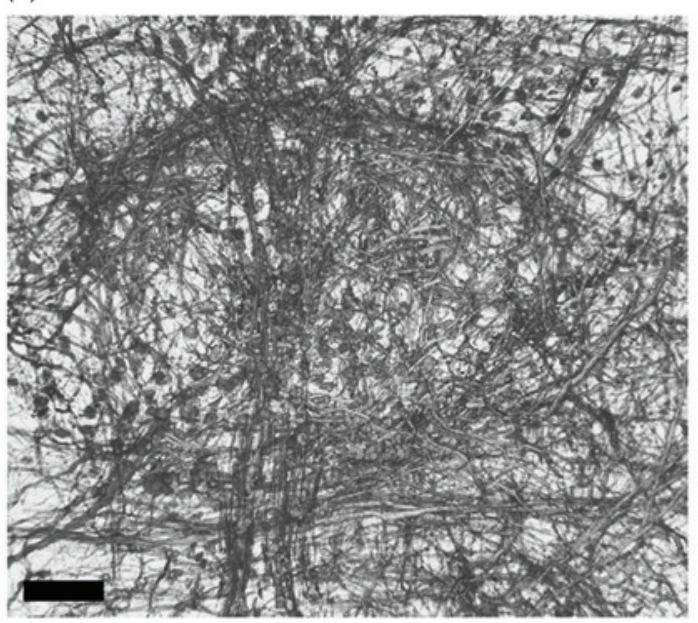

(b)

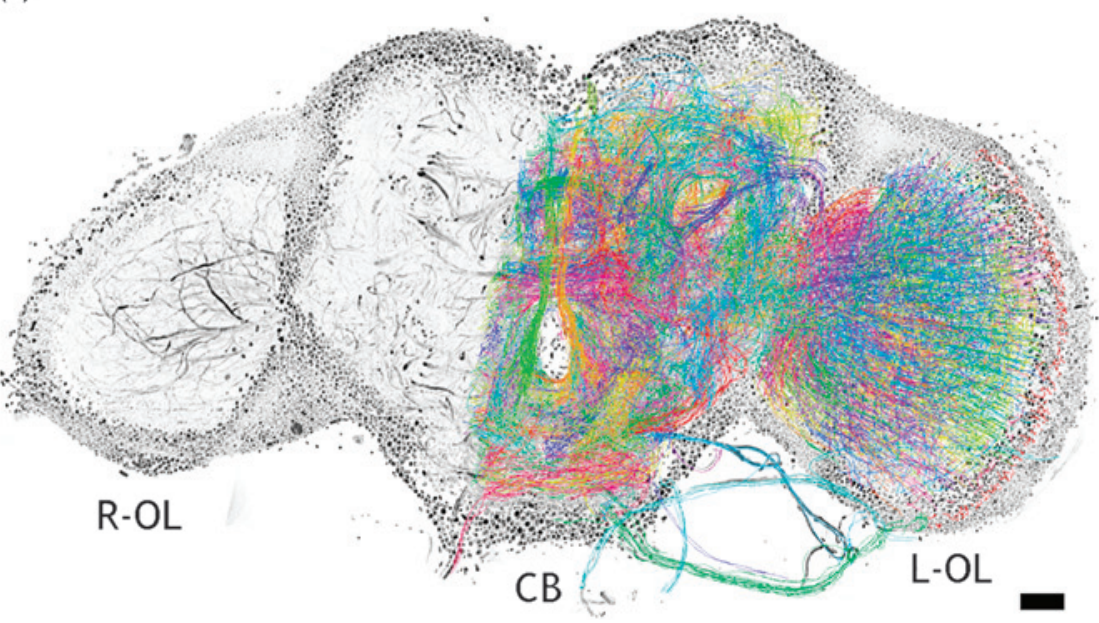

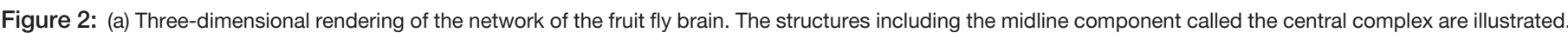

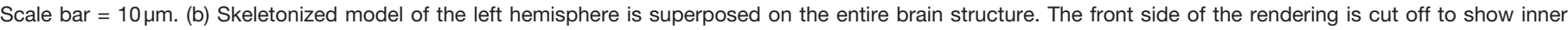
structures. Scale bar $=20 \mu \mathrm{m}$. CB, central brain; OL, optic lobe. 


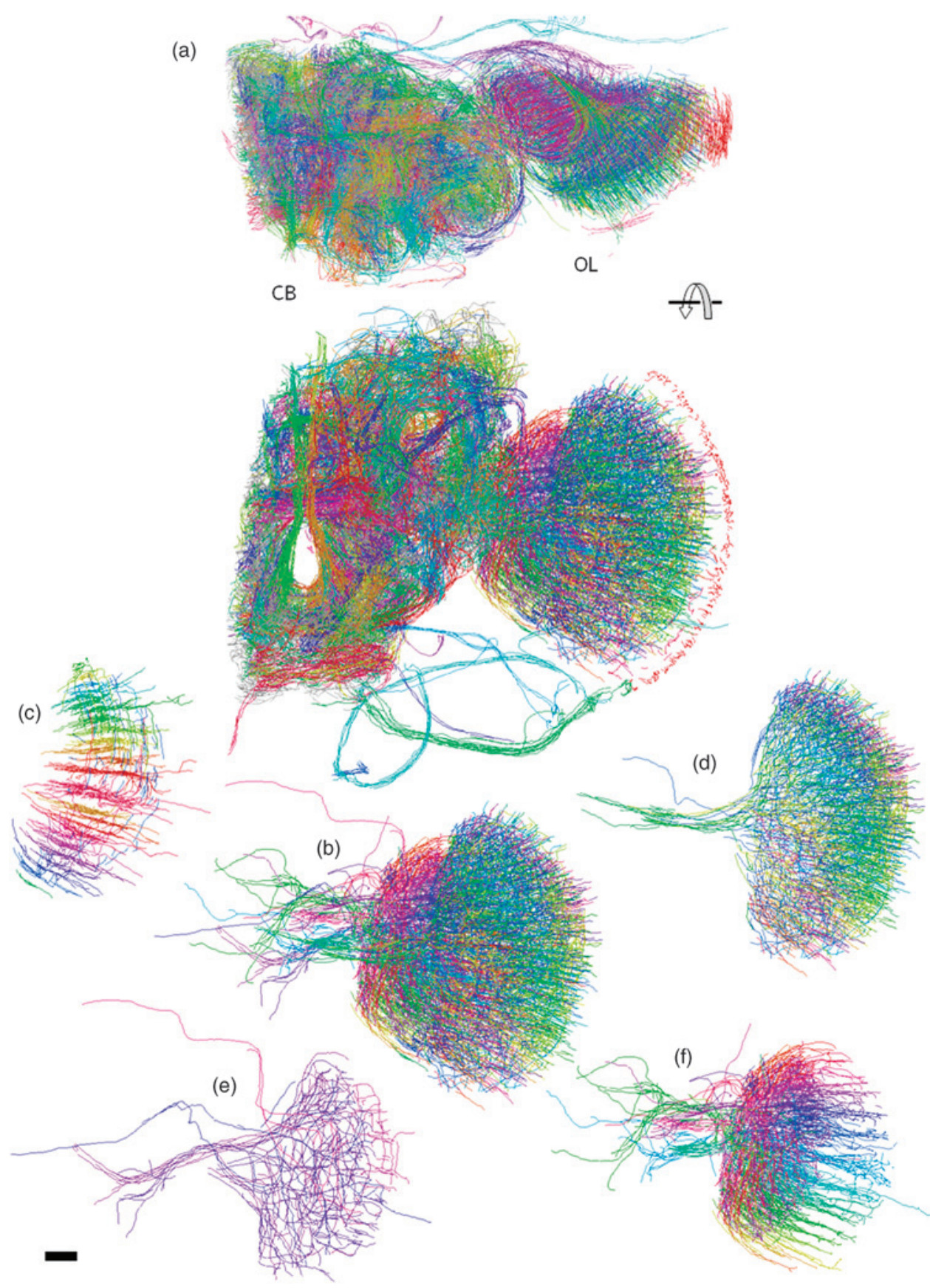

Figure 3: Skeletonized model of the left hemisphere of the fruit fly brain. The entire model (a) can be divided into CB and OL regions. The upper panel of (a) shows a side view with dorsal side toward the top. The lower panel of (a) shows a front view of the left hemisphere with the dorsal side toward the top The OL (b) can be further divided into the second optic chiasma (c), medulla (d), lobula plate (e), and lobula (f). Structural groups are color-coded. Scale bar $=20 \mu \mathrm{m}$.
X-ray tomographic microscopy with Fresnel zone plate (FZP) optics was performed at the BL37XU and BL47XU beamlines. In the BL37XU experiment, a FZP with an outermost zone width of $100 \mathrm{~nm}$ and diameter of $310 \mu \mathrm{m}$ was used as an X-ray objective lens to visualize structures at 160-200 $\mathrm{nm}$ resolution. In the BL47XU experiment, a FZP with an outermost zone width of $100 \mathrm{~nm}$ and diameter of $774 \mu \mathrm{m}$ was used as an X-ray objective lens to take images with a viewing field wider than $500 \mu \mathrm{m}$. Transmission images were recorded with a CMOS-based imaging detector
(Hamamatsu Photonics, Japan). The data acquisition conditions are summarized in Table 1.

$\mathrm{X}$-ray images were subjected to a convolution back-projection calculation using the RecView program (available from http://www.el.u-tokai.ac.jp/ ryuta/). The obtained tomographic cross sections were stacked in order to reconstruct a $3 \mathrm{D}$ image of the sample. Spatial resolution was estimated with 3D square-wave patterns [3].

Network analysis. In order to analyze the brain network, the structure of each neuron should be described by building a skeletonized model in the 3D image. The model was built by using a method like those used in crystallographic studies of macromolecular structures [1]. Because it takes considerable person-hours to manually build models of neuronal networks, an initial model was automatically built by machine interpretation of the 3D image [1,2]. First, large structures, such as cell body somas and blood vessels, were scanned to mask those regions from the subsequent tracing procedure. Cartesian coordinates of the large structures were also used for locating neuronal somas. Next, fibriform structures corresponding to dendrites and axons were searched by evaluating the gradient vector flow. The resultant coordinates with high fibriform scores were used as starting points to trace the neuronal processes using a Sobel filter [1]. This procedure was implemented with dedicated software to build models automatically $[1,2]$. The models obtained were manually examined and edited so as to assemble neuronal processes as neurons or to classify them into
3D structural groups.

\section{Results}

Fly brain network. Figure 2 a shows the $3 \mathrm{D}$ structure of the fruit fly brain visualized at the BL37XU beamline. The spatial resolution of this image was estimated to be $160-200 \mathrm{~nm}$. Neuronal processes were clearly visualized as network structures. In order to analyze the overall network, a skeletonized model was built using a 3D image obtained at BL47XU (Figure 2b). The obtained model consists of neuronal processes with a total length of $378 \mathrm{~mm}$ in a volume of $0.220 \times 0.328 \times 0.314 \mathrm{~mm}^{3}$. 


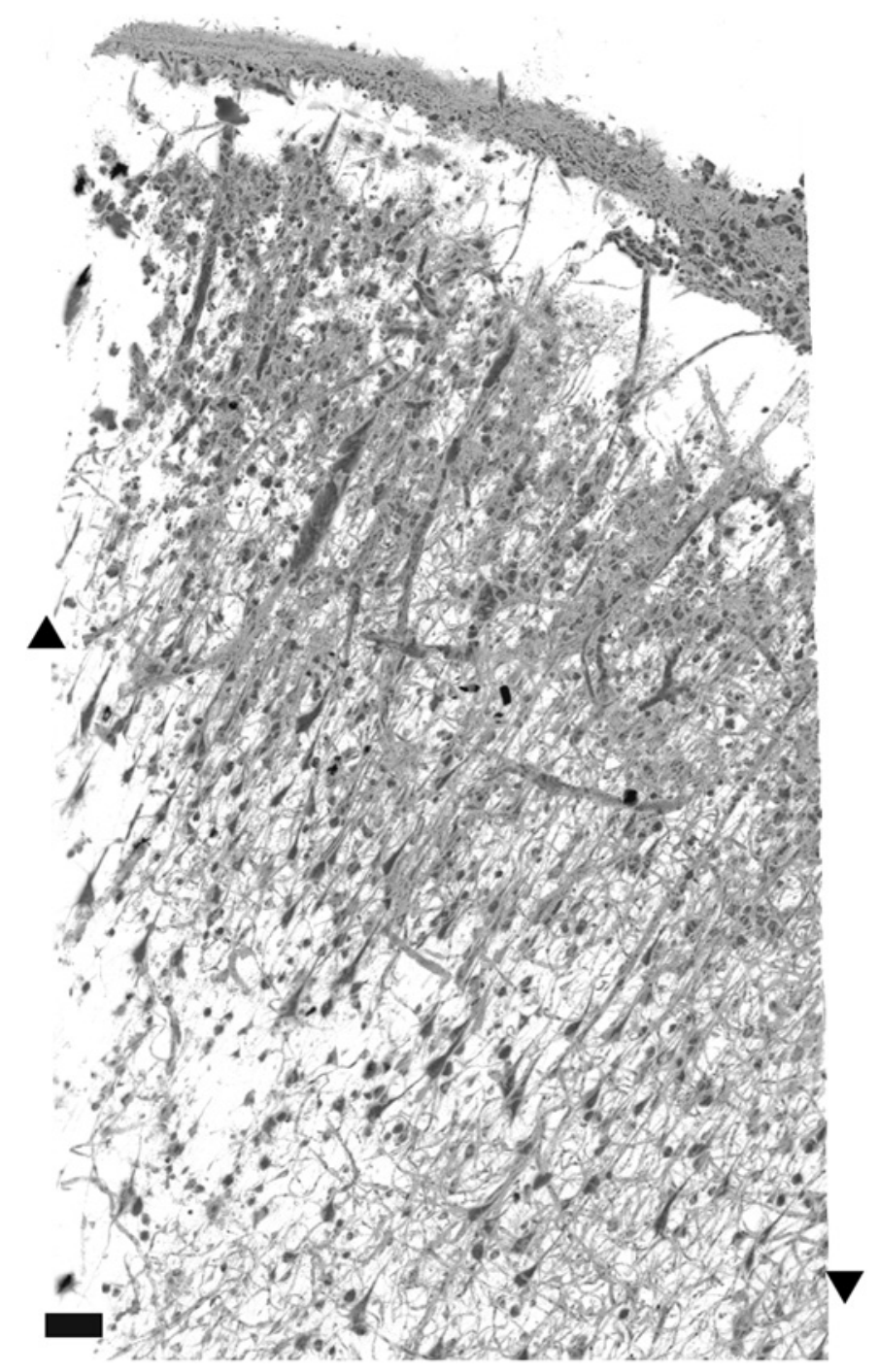

Figure 4: Three-dimensional structure of human frontal cortex tissue. X-ray linear attenuation coefficients were rendered from $9 \mathrm{~cm}^{-1}$ (white) to $112 \mathrm{~cm}^{-1}$ (black). Arrowheads indicate the internal pyramidal layer. Scale bar $=100 \mu \mathrm{m}$.

The network constituents were classified into groups on the basis of their 3D structures [2]. The classified model (Figure 3) allowed us to extract anatomical segments by specifying neuronal processes in a group-by-group manner. For example, the lobula plate (Figure 3e) located at the posterior end of the optic lobe can be distinguished from the other structures by its location and structures specific to the lobula plate. Neurons of the lobula plate were characterized by their fan-shaped ramification. Such structures of the lobula plate can be extracted from the model by specifying corresponding structural groups.

Figures $3 b-3 f$ show the organization of the optic lobe network, which is responsible for visual information processing. Neuronal processes of the medulla and second optic chiasma, which are proximal to the compound eye, exhibit periodical structures corresponding to repeated units of photoreceptors. On the opposite side of the optic lobe, neuronal processes are assembled into several tracts pointed toward central brain regions. These structures represent information paths from the inputs on the eye to the integrative process in the central brain.
Refinements of these models by using the high-resolution data should reveal finer 3D aspects of the fly brain.

Human brain circuits. Figures 4 and 5 show the structures in the human frontal cortex tissues. The $3 \mathrm{D}$ images of a block sample with dimensions of $1.4 \mathrm{~mm} \times 1.4 \mathrm{~mm} \times 8.3 \mathrm{~mm}$ are so complicated (Figure 4) that they cannot be comprehended at a glance, although pyramidal neurons arranged in a soma layer, called the internal pyramidal layer, can be seen in the image. A smaller sample with dimensions of $0.30 \mathrm{~mm} \times 0.35 \mathrm{~mm} \times$ $2.4 \mathrm{~mm}$ was also visualized with tomographic microscopy and subjected to model building. Skeletonized models of neurons (Figure 5a) were built by tracing the neuronal processes and assembling them into neurons. Although it is still difficult to comprehend the entire models, any of the neurons composing them can be extracted (Figure 5b). Because the models are described in terms of 3D Cartesian coordinates, the distances between the neuronal processes or somas can be directly calculated from the coordinates. This enabled us to determine individual neuronal circuits by analyzing the positional relationships of the neurons [1]. The pair of neurons shown in Figure 5b connect their inputs and outputs to each other to form a feedback loop (Figure 5c). In electronics, a similar feedback circuit composed of transistors is known as an astable multivibrator (Figure $5 \mathrm{~d}$ ), which generates a string of pulses. The refractory period of neurons prevents repetitive action potentials and hence poses a limit on the firing interval. However, a delay through a loop composed of a number of neurons allows recovery from the refractory period of a few milliseconds, resulting in oscillations of the loop circuit. Because such feedback loops are formed if a number of neurons are connected to each other, the loop circuit should be one of the canonical structures of human brain circuits.

\section{Discussion}

Three-dimensional structures of neuronal networks of human and fruits fly brains were visualized with X-ray tomographic microscopy $[1,2]$. The obtained structures were analyzed by building skeletonized models of neurons. This quantitative description of 3D networks in Cartesian coordinate space enabled examination of the functional mechanisms of the obtained brain networks.

The functional architecture of the visual cortexes of cat and monkey brains has been incorporated into artificial neural networks [4]. This neural network, called a convolutional neural network, has been applied to image recognition tasks and has outperformed other computing methods designed without reference to the visual cortex architecture [4]. The present study illustrated some of the circuits of the human frontal cortex, which is responsible for higher brain functions. These circuits should provide a basis for cognitive computing to deal with complicated problems that have not been able to be handled without human intervention.

The psychological individuality of human beings is ascribable to the individuality of their brains. Therefore, psychological characters reside in the neuronal circuits of the brain. Differences between neuronal circuits should be observable not only in healthy individuals, but also between patients with brain disorders and healthy controls. Such differences can be clues to improving the treatments of brain disorders. Because our knowledge of the circuits in the human brain is still limited, drugs for treating 
(a)

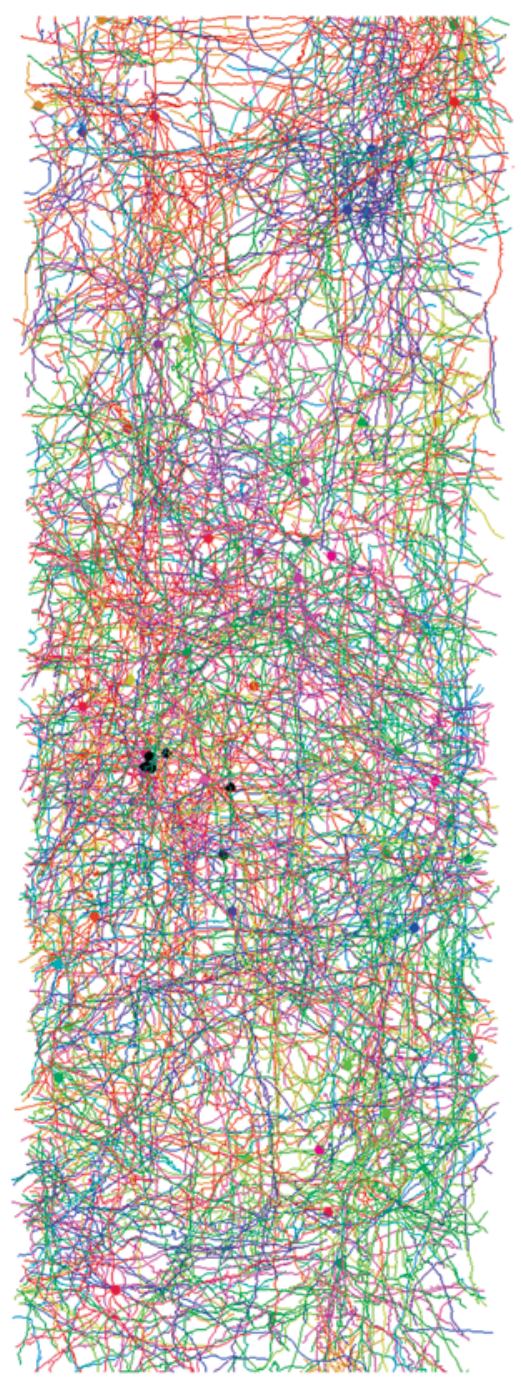

(b)

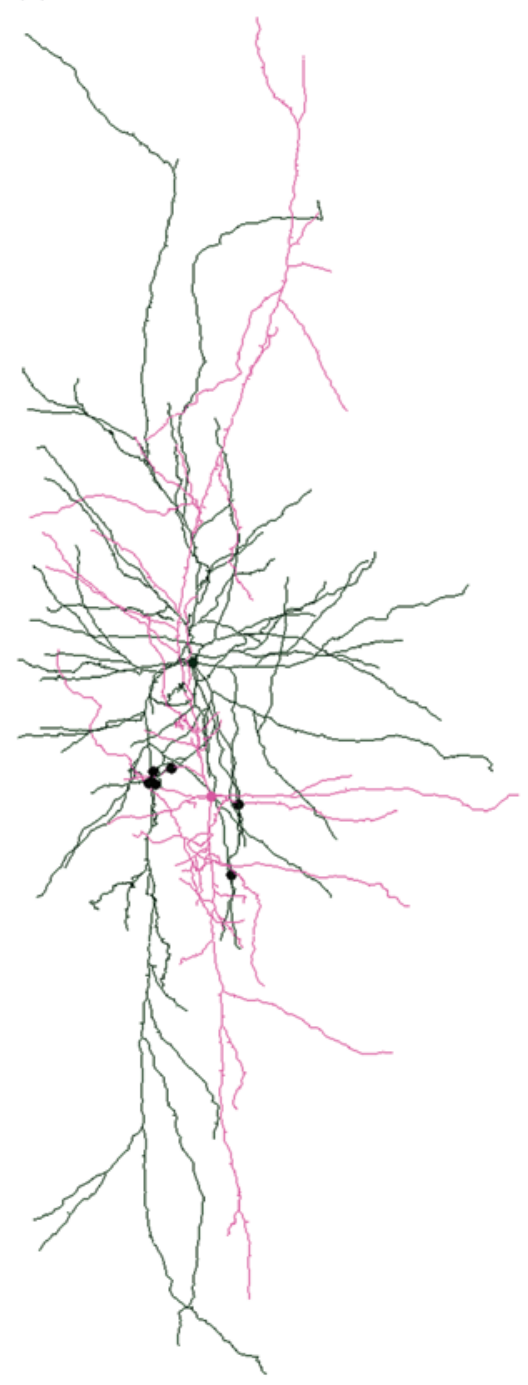

(c)

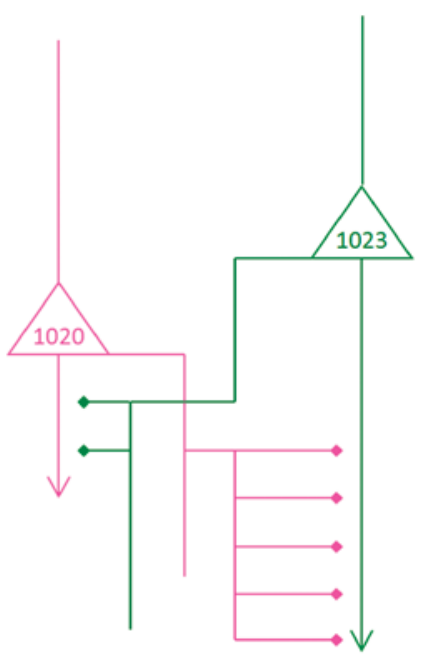

(d)

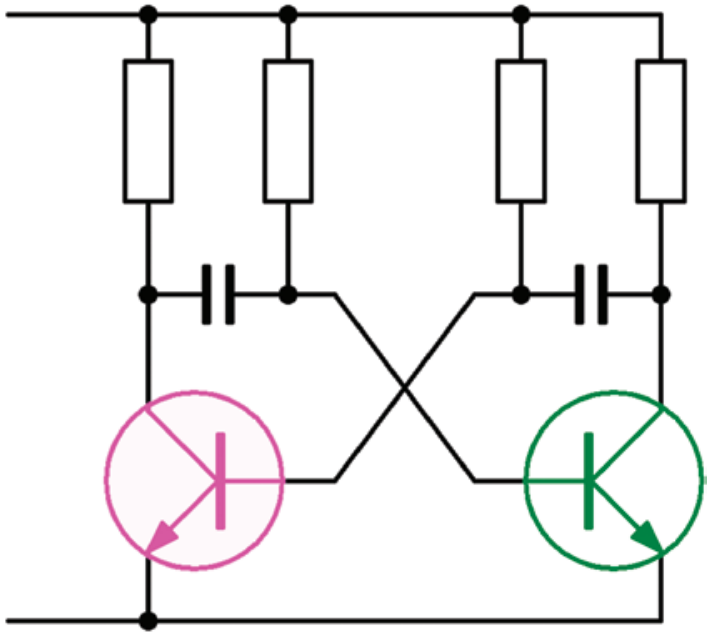

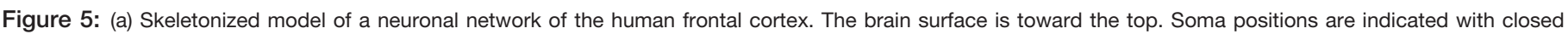

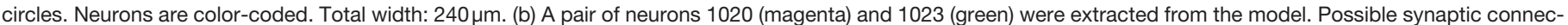

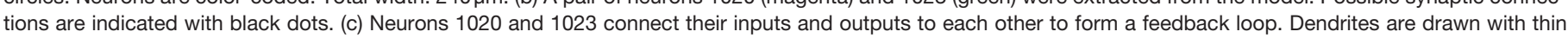

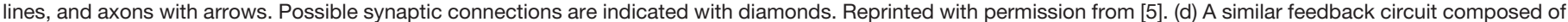
transistors is known as an astable multivibrator.

brain disorders are being administrated without a complete understanding of their mechanisms of action on the circuits of the brain. Better diagnosis, prevention, and treatment of brain disorders should thus be possible by analyzing brain networks and identifying neuronal circuits responsible for individual brain disorders.

\section{Conclusion}

Neurons make up a 3D network in brain tissue. This article reports on the $3 \mathrm{D}$ structures in human and fruit fly brain tissues as measured with a microscopic version of medical computed tomography (CT) called X-ray tomographic microscopy (or microtomography or micro-CT). The obtained structures were analyzed by building skeletonized models of neurons. The resulting quantitative description of the $3 \mathrm{D}$ networks provided information about the functional mechanisms of the brain.

\section{Acknowledgments}

This work was supported in part by Grants-in-Aid for Scientific Research from the Japan Society for the Promotion of Science (nos. 25282250 and 25610126). The synchrotron radiation experiments were performed at SPring- 8 with the approval of the Japan Synchrotron Radiation Research Institute (JASRI) (proposal nos. 2008B1261, 2013B0034, 2013B0041, 2014B1083, 2014B1096, and 2015A1160).

\section{References}

[1] R Mizutani et al., Cereb Cortex 20 (2010) 1739-48.

[2] R Mizutani et al., J Struct Biol 184 (2013) 271-9.

[3] R Mizutani et al., Nucl Instrum Meth A 621 (2010) 615-9.

[4] Y Bengio, Foundations and Trends in Machine Learning 2 (2009) 1-127.

[5] R Mizutani et al., KENBIKYO 49 (2014) 222-25. 


\section{MICROSCOPES}

I N T E R N A T O N A L

\section{Isn't it about time you had your own Digital Microscope?}

Now you can with the portable, affordable uScopeMXII!

The uScopeMXII is a small digital desktop microscope you can use in your workplace or home office. It captures images from standard glass slides and sends them to your PC.

You can interactively browse slides with full control of focus, image processing, and location. You can also scan regions of interest creating fully focused image sets.

The industry-standard USB interface makes it simple to plug in and start capturing images. It easily interfaces with your desktop or laptop PC and allows you to view and capture slide images in a wide variety of environments.

The uScopeMXII is manufactured in the United States.

\section{Features and Benefits}

- Overview and Objective Cameras The uScopeMXII has an objective camera for scanning and an overview camera for navigating.

- Automatic Focus

Images are automatically focused using configurable focus algorithms.

\section{- Portability}

At a weight of about $5 \mathrm{lbs}$., the uScopeMXII is highly portable.

- Easy to Use

The uScope Navigator software simplifies scanning and browsing.

- Self-Contained

The uScopeMXII is self-contained and includes the electronics, cameras, stage, and optics in a device about the size of a large external disk drive.

\section{- Full Imaging Control}

User filters provide complete control over image processing and correction.

Microscopes International, LLC 555 Republic Drive, Suite 119

Plano, TX 75074-5498

Phone +1-214-785-2058

FAX +1-214-785-2138

Emailssales@uscopes.com support@uscopes.com

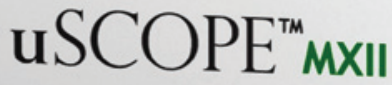

- POWER

- STATUS

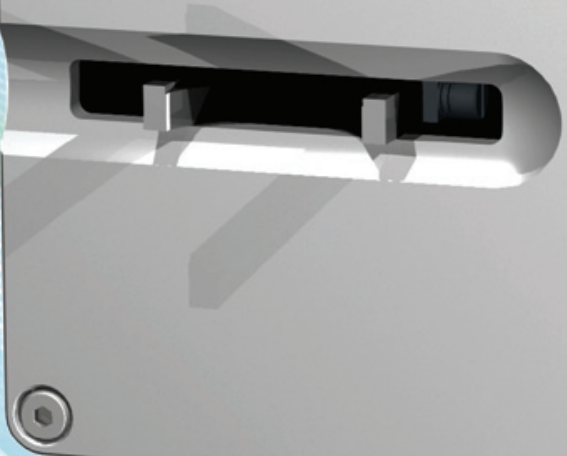

Call us today for a quote or to schedule a demonstration 